Alfred H.J. Kim, MD, PhD

Division of Rheumatology, Department of Medicine,

Washington University School of Medicine, St. Louis, MO
Xiaobo Wu, MD

Division of Rheumatology, Department of Medicine,

Washington University School of Medicine, St. Louis, MO
John P. Atkinson, MD

Division of Rheumatology, Department of Medicine,

Washington University School of Medicine, St. Louis, MO

\title{
The beneficial and pathogenic roles of complement in COVID-19
}

\section{Posted October 20, 2020}

\section{ABSTRACT}

We briefly summarize the complement system and its functions in immunity and disease. We present data supporting the requirement of complement to resolve COVID-19, and discuss how complement overactivation later in severe disease could drive multiorgan damage characteristic of fatal COVID-19.

\section{INTRODUCTION}

The coronavirus disease 2019 (COVID-19) pandemic has created a healthcare crisis unmatched in our lifetime. Caused by severe acute respiratory syndrome coronavirus-2 (SARS-CoV-2), more than 28 million cases have been reported worldwide, including more than 7.6 million in the United States (https://coronavirus.jhu.edu/map.html). This disease has resulted in at least 1 million deaths globally, and more than 213,000 of these in the United States.

COVID-19 has a widely varied presentation (Table 1 ), being asymptomatic in $20 \%$ to $40 \%$ of cases, a common cold or influenza syndrome in $30 \%$ to $70 \%$, or a severe life-threatening disease in less than $10 \%$. Severe disease is characterized by respiratory failure, a hyperinflammatory internal milieu, and multiorgan thrombotic complications.

The complement system has recently gained interest as a contributing player to certain pathogenic features of severe COVID-19 infection, particularly relative to thrombotic microangiopathy (TMA). Autopsy reports have also identified complement-mediated pathologic features consistent with manifestations of TMA such as endothelial cell abnormalities and

Dr. Kim has disclosed consulting for Alexion Pharmaceuticals. Dr. Wu and Dr. Aktinson have nothing to disclose.

The statements and opinions expressed in COVID-19 Curbside Consults are based on experience and the available literature as of the date posted. While we try to regularly update this content, any offered recommendations cannot be substituted for the clinical judgment of clinicians caring for individual patients.

doi:10.3949/ccjm.87a.ccc065 complement fragment deposition in the lungs, skin, and kidney. ${ }^{1,2}$ In addition, recent transcriptional and genetic loci studies have provided further evidence for the involvement of the complement system in COVID-19. Indeed, therapies targeting complement components demonstrate preliminary success in treating severe COVID-19. ${ }^{3-5}$ But what has been somewhat ignored is the antiviral contribution of the complement system in a response to COVID-19, particularly early in the infection.

Herein, we briefly summarize the complement system and its functions in immunity and disease, presenting data supporting both the requirement of complement to resolve COVID-19, and how complement overactivation later in severe disease could drive multiorgan damage characteristic of fatal COVID-19. For the sake of brevity, we have primarily referenced a limited number of reviews and original reports with a focus on the complement system..$^{2-13}$

\section{THE COMPLEMENT SYSTEM IN IMMUNITY AND DISEASE}

The complement system is a proteolytic cascade initiated by three pathways (classical, alternative, and lectin), each uniquely triggered to generate a potent, highly regulated, innate immune response and to set the stage for a prompt and decisive adaptive immune response: 1) membrane perturbation featuring $\mathrm{C} 4 \mathrm{~b}$ and C3b-mediated opsonization/phagocytosis, as well as a lytic process mediated by the membrane attack complex (MAC, C5b-9), and 2) generation of a proinflammatory state largely mediated via the anaphylatoxins C3a and C5a. Using these same two general mechanisms, the complement system also facilitates the clearance of apoptotic material and cellular debris. Further, intracellular complement activation enables cells to modulate metabolic pathways and thereby regulate immune responsiveness. ${ }^{6}$

In systemic lupus erythematosus (SLE) and related autoimmune diseases, immune complexes generated 
TABLE 1

Clinical classification of COVID-19 infection, and the role of complement activation

\begin{tabular}{|c|c|c|}
\hline Disease severity & Percentage of cases & Features/role of complement activation \\
\hline \multirow[t]{2}{*}{ Asymptomatic } & $20 \%-40 \%$ & $\begin{array}{l}\text { Immune system shuts off the virus; no clinical signs or symptoms of } \\
\text { infection; immunity induced; " natural" vaccination }\end{array}$ \\
\hline & & Complement activation beneficial \\
\hline \multirow[t]{2}{*}{$\begin{array}{l}\text { Upper respiratory tract } \\
\text { (cold-like syndrome) }\end{array}$} & $30 \%-70 \%$ & $\begin{array}{l}\text { Virus replicates in upper-airway tissues leading to sore throat, } \\
\text { cough, fever, sneezing, altered taste and smell, "typical cold" syn- } \\
\text { drome; usually lasts } 2 \text { to } 5 \text { days; recovery in } 2 \text { to } 3 \text { weeks }\end{array}$ \\
\hline & & Complement activation beneficial $^{\mathrm{a}}$ \\
\hline \multirow[t]{2}{*}{ Moderately severe } & $5 \%-10 \%$ & $\begin{array}{l}\text { Viral load is greater; extension to deeper parts of the lung (pneu- } \\
\text { monia) and possibly to other tissues, with prominent inflammatory } \\
\text { picture }\end{array}$ \\
\hline & & Complement activation not beneficial \\
\hline \multirow[t]{4}{*}{ Severe } & $1 \%-5 \%$ a & $\begin{array}{l}\text { Heavy viral load; extension to extrapulmonary tissues, especially } \\
\text { endothelial cell populations; thromboembolic phenomena }\end{array}$ \\
\hline & & Virus is never adequately controlled ${ }^{\mathrm{b}}$ \\
\hline & & $\begin{array}{l}\text { Virus eventually controlled and eliminated, but often with residual } \\
\text { effects }\end{array}$ \\
\hline & & Complement activation poor for host \\
\hline
\end{tabular}

an these patients, early in the course of the infection, immune enhancement may be indicated.

bIn these patients, dampening the inflammatory response could be advantageous, especially if the viral replication has been controlled.

by autoantibodies drive type II and III hypersensitivity reactions that leverage primarily the classical pathway activation to initiate destructive inflammatory responses. ${ }^{2,7}$ Interestingly, the failure to clear apoptotic debris, such as in complete classical pathway component $\mathrm{C} 1 \mathrm{q}, \mathrm{C} 4$, or $\mathrm{C} 2$ deficiency, is strongly predisposing to SLE. ${ }^{8}$ In age-related macular degeneration and atypical hemolytic uremic syndrome, loss of function (commonly haploinsufficiency) of complement regulatory proteins or gain of function in complement activating components promotes excessive alternative pathway engagement, leading to retinal damage in age-related macular degeneration and microthrombi featuring endothelial injury in atypical hemolytic uremic syndrome. ${ }^{2,7}$

\section{EARLY CONTROL OF SARS-COV-2 LIKELY REQUIRES COMPLEMENT ACTIVATION}

In the early phase of the innate immune response in COVID-19, a robust antiviral response to SARSCoV-2 occurs in which the complement system plays an important role. Natural and cross-reacting antibodies and lectins trigger complement activation to destroy complement-coated virus and block viral entry. Mice infected with the related SARS-CoV virus generate numerous $\mathrm{C} 3$ activation products (C3a, C3b, iC3b, C3c, C3d) in the lung within a day. ${ }^{9}$ Infection of cells by SARS-CoV-2 may promote $\mathrm{C} 3$ activation, as suggested in a preprint publication demonstrating that the $\mathrm{N}$ protein of SARS-CoV-2 activated the mannan-binding lectin-associated serine protease (MASP-2). ${ }^{14}$ In another report utilizing an in vitro system, spike proteins 1 and 2 led to activation primarily of the alternative pathway on human cells. ${ }^{13}$ These types of innate immune responses coupled with a rapid adaptive response lead to either no clinical signs of infection or a common cold-like or influenza-like syndrome.

Viruses, as might be expected, have developed evasion tactics to limit complement activation. ${ }^{10}$ For example, poxviruses express a protein that is structurally and functionally similar to human complement regulatory proteins. Other pathogens "hijack" our own regulators to protect themselves. Flaviviruses produce proteins that down-regulate the complement system's activating capabilities. It remains unknown whether coronaviruses also possess antiviral activity. 


\section{INADEQUATE COMPLEMENT ACTIVATION CHARACTERIZES LATE COVID-19}

Inadequate control of SARS-CoV-2 replication early in infection will lead to a heavier viral load, and an exuberant immune response will naturally follow, including complement activation. A robust proinflammatory cytokine (interleukin 6 , interleukin 8) response has been observed in severe infections (often associated with a so-called cytokine release or cytokine storm syndrome). This result could be secondary in part to the viral damage to infected tissues (even though viral replication is halted), but an exuberant immune response to the accumulating debris occurs. $3,4,9$

Further, data implicating a prominent role for the complement system in severe COVID-19 came from early clinicopathologic studies. C5b-9, C4d, and MASP-2 deposition was noted in affected tissues, including the skin in those with reticuliform and purpuric lesions and the lung microvasculature in patients who died due to respiratory failure. ${ }^{1} \mathrm{C} 5 \mathrm{~b}-9$ deposition was also observed in the microvasculature in unaffected deltoid skin in 21 out of 23 hospitalized COVID-19 patients, which colocalized with the SARS-CoV-2 spike protein and ACE2 in the endothelial cells. ${ }^{15}$ While COVID-19 lung pathology is distinct from typical acute respiratory distress syndrome, complement activation within the lung has commonly been observed in acute respiratory distress syndrome, suggesting a possible common etiology. ${ }^{12}$ Complement activation fragments have also been observed to be elevated in serum of patients with severe COVID-19.2 Additionally, histopathologic features with similarities to other complement-mediated diseases indicate that complement deposition is a pathologic actor in severe COVID-19. Endothelial cell abnormalities such as cellular swelling with foamy degeneration in the setting of TMA have been observed in numerous organs, consistent with C5b-9-mediated injury and a hypercoagulable state. These data are consistent with the murine model of SARS-CoV, in which C3-deficient mice experienced less lung inflammation and injury. ${ }^{9}$

One mechanism by which complement may be overactivated in severe COVID-19 lies in the interplay between neutrophils and complement. Activated neutrophils generate extracellular chromatinrich structures called neutrophil "traps," which bind pathogenic material. Neutrophil traps promote alternative pathway activation as they contain $\mathrm{C} 3$, factor $\mathrm{B}$, and properdin. Exuberant neutrophil activation and formation of neutrophil traps have been noted in patients with severe COVID-19.2

\section{CONCLUSION}

It is clear that complement activation occurs in COVID-19 and likely serves different roles depending on the time course of the infection (Table 1). Early clinical management of COVID-19 needs not only to support innate immunity including complement activation against the virus, but also to curtail subsequent potential damage to the tissues or organs in the severe syndrome (assuming viral replication has been largely stopped). Early on, complement is required to assist antiviral responses, such as clearing of complementcoated virions. Later, in the subset of patients with severe COVID-19 disease (too much virus or tissue damage), complement drives several of the observed pathogenic features. This has substantial implications in the use of complement therapeutics.

Scenarios in which timing of complement activation needs to be considered include the transfusion of convalescent plasma, which will form immune complexes with the intact virus (a good effect) but also with viral remnants and damaged tissues, potentially exacerbating the situation. Similarly, the use of steroids (or other immune-inflammatory suppressors) could inhibit the immune response to COVID-19. Here again, the timing of the administration of these immunosuppressive medications could be critical, as is knowledge relative to the viral load and replicating capability.

Thus, the timing of administration may lead to poor or improved outcomes. On one hand, complement inhibition in early disease may lead to worse clinical outcomes or poor protective responses, while in late disease, complement inhibition may improve outcomes. Indeed, preliminary reports of complement inhibition in severe COVID-19 have been encouraging. For example, a novel inhibitor to C3, AMY101, which prevents C3 cleavage to C3a and C3b, has anecdotal success in severe COVID-19. This led to an active phase II trial in COVID-19 patients with respiratory distress. ${ }^{3,5}$ Furthermore, monoclonal antibodies targeting C5 activation (eculizumab, ravulizumab) have also demonstrated efficacy in COVID19, with several active phase III clinical trials currently recruiting. 4.5 Additional answers will emerge as these and other clinical trials report their results, providing an improved understanding of how to best manipulate the complement system in COVID-19. 


\section{REFERENCES}

1. Magro C, Mulvey JJ, Berlin D, et al. Complement associated microvascular injury and thrombosis in the pathogenesis of severe COVID-19 infection: a report of five cases. Transl Res 2020; 220:1-13. doi:10.1016/j.trsl.2020.04.007

2. Java A, Apicelli AJ, Liszewski MK, et al. The complement system in COVID-19: friend and foe? JCI Insight 2020; 5(15):e140711. doi:10.1172/jci.insight.140711

3. Mastaglio S, Ruggeri A, Risitano AM, et al. The first case of COVID19 treated with the complement C3 inhibitor AMY-101. Clin Immunol 2020; 215:108450. doi:10.1016/j.clim.2020.108450

4. Kulasekararaj AG, Lazana I, Large J, et al. Terminal complement inhibition dampens the inflammation during COVID-19. Br J Haematol 2020; 190(3):e141-e143. doi:10.1111/bjh.16916

5. Mastellos DC, Pires da Silva BGP, Fonseca BAL, et al. Complement C3 vs $C 5$ inhibition in severe COVID-19: early clinical findings reveal differential biological efficacy. Clin Immunol 2020; 220:108598. doi:10.1016/j.clim.2020.108598

6. Liszewski MK, Elvington M, Kulkarni HS, Atkinson JP. Complement's hidden arsenal: new insights and novel functions inside the cell. Mol Immunol 2017; 84:2-9. doi:10.1016/j.molimm.2017.01.004

7. Liszewski MK, Java A, Schramm EC, Atkinson JP. Complement dysregulation and disease: insights from contemporary genetics. Annu Rev Pathol 2017; 12:25-52. doi:10.1146/annurev-pathol-012615-044145

8. Macedo AC, Isaac L. Systemic lupus erythematosus and deficiencies of early components of the complement classical pathway. Front Immunol 2016; 7:55. doi:10.3389/fimmu.2016.00055

9. Gralinski LE, Sheahan TP, Morrison TE, et al. Complement activation contributes to severe acute respiratory syndrome coronavirus pathogenesis. mBio 2018; 9(5): e01753-18. doi: 10.1128/mBio.01753-18

10. Stoermer KA, Morrison TE. Complement and viral pathogenesis. Virology 2011; 411(2):362-373. doi: 10.1016/j.virol.2010.12.045

11. Vabret N, Britton GJ, Gruber C, et al. Immunology of COVID-19: current state of the science. Immunity 2020; 52(6):910-941. doi: 10.1016/j.immuni.2020.05.002

12. Robbins RA, Russ WD, Rasmussen JK, Clayton MM. Activation of the complement system in the adult respiratory distress syndrome. Am Rev Respir Dis 1987; 135(3):651-658. doi:10.1164/ arrd.1987.135.3.651

13. Yu J, Yuan X, Chen H, Chaturvedi S, Braunstein EM, Brodsky RA. Direct activation of the alternative complement pathway by SARSCoV-2 spike proteins is blocked by factor D inhibition. Blood 2020; blood.2020008248. doi: 10.1182/blood.2020008248.

14. Gao T, Hu M, Zhang X, et al. Highly pathogenic coronavirus N protein aggravates lung injury by MASP-2-mediated complement overactivation. medRxiv 2020. doi:https://doi.org/10.1101/2020.03.29.20 041962.

15. Magro C, Mulvey JJ, Laurence J, et al. Docked SARS-CoV-2 Proteins within the Cutaneous and Subcutaneous Microvasculature and their Role in the Pathogenesis of Severe COVID-19. Human Path 2020; Online 12 October 2020. doi:10.1016/j.humpath.2020.10.002

Correspondence: John P. Atkinson, MD, Washington University School of Medicine, Division of Rheumatology, 660 South Euclid Ave, Campus Box 8045, Saint Louis, MO 63110; j.p.atkinson@wustl.edu 